\title{
MODELAGEM NUMÉRICA CONTÍNUA E EQUIVALENTE CONTÍNUA PARA AVALIAR ESTABILIDADE DE TALUDES SUSCEPTÍVEL A TOMBAMENTO
}

\author{
D. S. $\operatorname{COSTA}^{1}$, R. BUGS ${ }^{2}$, M. C. A. FEITOSA ${ }^{1}$, A. C. ZINGANO ${ }^{2}$ \\ ${ }^{1}$ Instituto Federal de Educação, Ciência e Tecnologia do Rio Grande do Norte \\ ${ }^{2}$ Universidade Federal do Rio Grande do Sul \\ costa.daniel@ifrn.edu.br*
}

Artigo submetido em abril/2016 e aceito em maio/2016

DOI: 10.15628/holos.2016.4367

\section{RESUMO}

O estudo da estabilidade de taludes nas bancadas é fato notório e indispensável para eficiência das operações e garantia da segurança em minas a céu aberto. A presença de famílias de descontinuidades, em especial nas cavas de grandes profundidades, pode indicar dentre outras formas de ruptura, instabilidade por tombamento. Este trabalho foi realizado para comparar e discutir os modelos utilizados no entendimento das formas de ruptura por tombamento em uma mina de calcário. Por meio de dados de campo e modelagem por elementos finitos, foram construídos dois modelos: no primeiro, o maciço é tratado como um corpo único, isto é, com as descontinuidades naturais do processo de formação da rocha e caracterizado por meio de classificação geomecânica. Já no segundo modelo, o corpo também é considerado contínuo, mas com as descontinuidades inseridas. Esta modelagem numérica foi realizada com auxílio do software Phase $^{2}$ V.8 da Rocscience. Os resultados revelam semelhanças entre os fatores de segurança em níveis de estabilidade, sendo que no modelo contínuo (mas com descontinuidades inseridas) se observou de forma mais clara as tensões cisalhantes induzidas nas descontinuidades durante o movimento de tombamento.

PALAVRAS-CHAVE: Tombamentos, scanline, modelos numéricos, elementos finitos, modelo contínuo.

\section{MODELING NUMERICAL EQUIVALENT CONTINUOUS AND CONTINUING TO EVALUATE SLOPE STABILITY SUSCEPTIBLE THE TIPPING}

\begin{abstract}
The study of slope stability in benches is indeed remarkable and indispensable for the efficiency of operations and safety in open pit mines. The presence of discontinuities families, especially in great depths can indicate, among other forms, toppling. This study was conducted to compare and discuss the models used in the understanding of the ways to rupture toppling in a limestone mine. Through the field data and modeling by finite elements, were constructed two models: in the
\end{abstract}

first, the massive is treated as a single body and characterized by means of geomechanical classification. In the second model, the body is also considered continuous, but with inserted discontinuities. The results reveal similarities between the safety factors in stability levels, though in the continuous model (but with inserted discontinuities) was observed more clearly the shear stresses induced in the discontinuities during movement of toppling.

KEYWORDS: Toppliing, scanline, numerical modeling, finite elements, contínuous model. 


\section{INTRODUÇÃO}

Uma das maiores preocupações nas minerações a céu aberto são as condições da estabilidade dos taludes, uma vez que taludes instáveis podem comprometer a segurança das operações de lavra e proporcionar aumento de custos. Em cavas onde a profundidade está relativamente grande, acima de 100 metros, taludes globais podem estar propícios a problemas de instabilidade devido ao aumento da heterogeneidade das famílias de descontinuidades.

O problema aumenta quando há várias famílias de descontinuidades presentes no maciço e principalmente quando há possibilidades de rupturas por tombamento. Diante de tantas variáveis e riscos à segurança, a solução do problema não deve ser tratada por simples análises de equilíbrio limite.

Embora estudos relacionados a tombamentos tenham evoluído desde o método proposto por Goodman e Bray (1976), ainda há grande dificuldade, principalmente na comunidade mineira, em entender os mecanismos que controlam rupturas por tombamento e definir fatores de segurança a partir dos vários métodos analíticos disponíveis.

O objetivo deste trabalho é apresentar uma análise de ruptura por tombamento a partir do mapeamento das descontinuidades realizadas por Scanlines, em uma mina de calcário localizada no município de Pinheiro Machado - RS. As análises cinemáticas identificaram em uma das regiões da cava vulnerabilidade a este tipo de ruptura. As condições de estabilidade dos taludes, bem como simulações do movimento de ruptura, foram avaliadas por meio de modelagem numérica por elementos finitos com auxílio do software Phase ${ }^{2}$ V.8, da Rocscience.

Segundo Hoek et al (2002), análises relacionadas a movimento de tombamento podem ser tratadas por equilíbrio limite, no entanto estas fornecem apenas um fator de segurança, desconsiderando outros fatores como a forma que ocorre a ruptura. Neste caso, modelos numéricos são mais eficientes para tais problemas.

\section{REVISÃO BIBLIOGRÁFICA}

De acordo com a geologia estrutural e orientação das descontinuidades, Goodman e Bray (1976) classificaram as formas de tombamento em três grupos: i) blocos, ii) flexural e iii) blocoflexural. Baseando-se nessa divisão, vários autores sugeriram métodos analíticos para tratar problemas de instabilidade relacionado a movimentos de tombamento (Zanbak, 1983; Aydan e Kawamoto, 1992; Adhikary, Dyskin e Jewell, 1996; Adhikary et al., 1997; Bobet, 1999; Sagaseta, Sànchez e Cañizal, 2001).

Rupturas por tombamento, independentemente do grupo, ocorrem devido a um processo de instabilidade que é controlado pela frequência, orientação e parâmetros de resistência ao cisalhamento das descontinuidades (Zanbak, 1983). De acordo com Bobet (1999), esse tipo de ruptura pode ocorrer em todos os tipos de maciços desde que as descontinuidades possuam atitudes (dip e dip direction) favoráveis a ruptura.

Recentemente, Sagaseta Sànchez e Cañizal (2001) e Liu, Jaksa e Meyers (2009) sugeriram métodos analíticos para solucionar problemas relacionados a tombamento quando as famílias de 
descontinuidades tornam-se estruturas pequenas se comparado ao tamanho do talude. Tais métodos tratam o talude como várias regiões contínuas, mas não incorporam outros parâmetros tais como os citados em Pitchard e Savigny (1990) que são: poro-pressão e condições geológicas. Amini, Majdi e Veshadi (2012) também sugeriram metodologias para analisar tombamento por método analítico, mas este método, assim como os demais, não incorpora no problema várias famílias de descontinuidades.

Goodman e Bray (1976) propuseram que a condição cinemática favorável para tombamento ocorre quando a Equação (1) for satisfeita, onde $\theta$ é o ângulo da face do talude, $\phi j$ e $\delta$ são o ângulo de atrito e o mergulho das descontinuidades, respectivamente. $O$ fator de segurança pode ser determinado por essa metodologia (Udson e Harrison, 1997), mas esse método fornece apenas uma indicação de possibilidade de movimento flexural e não considera os mecanismos que controlam o escorregamento entre as camadas (Adhikary et al., 1997). Atualmente, este método é usado como uma análise preliminar de instabilidade por tombamento.

$\theta \geq 90^{\circ}+\phi j-\delta$

A estimativa da resistência de maciços é obtida por meio de critérios de ruptura, que é um fator que liga a resistência de amostras da matriz rochosa à resistência do maciço. Na prática, de acordo com Hoek et al (2002), a maioria dos projetos de estabilidade de taludes utilizam critérios de ruptura para estimar as propriedades de resistência da rocha e das descontinuidades. No caso de maciços, as propriedades de resistência destas estruturas, homogêneas e isotrópicas, são estimadas por meio do critério de ruptura e classificação geomecânica de Hoek-Brown.

Modelos numéricos por elementos finitos foram utilizados por vários autores para solucionar problemas relacionados a tombamentos (Burman, Trolope e Philip, 1975; Pritchard, 1989; Santos, 2009; Pereira, 2012). A aplicação desses métodos tiveram o objetivo de analisar os deslocamentos induzidos pelo processo de deformação a partir do estado de tensão no modelo.

Elementos finitos é um método que consiste na discretização da geometria de um problema em elementos que interagem de modo a verificar as deformações sofridas nos nós quando submetido às tensões (Brady e Brown, 2004). A solução do modelo ocorre por meio de matrizes que relaciona os estados de tensão e deformação, como demonstrada pela Equação 2. Onde K é a matriz de rigidez; U é a matriz de deformação; e F é a matriz de tensão aplicada no modelo.

$[\mathrm{K}]\{\mathrm{U}\}=\{\mathrm{F}\}$

A vantagem desse tipo de modelagem, no estudo das condições de rupturas por tombamento, é a capacidade de incluir informações geológicas para manter a heterogeneidade do maciço e por tratar, de forma mais realística, algumas limitações encontradas em métodos por equilíbrio limite tais como: poro-pressão, efeitos sísmicos, condições geológicas e geométricas (Pitchard e Savigny, 1990).

Embora o Phase2 V.8 faça modelagens tratando problemas como meios contínuos, ele tem capacidade para discretizar problemas inserindo descontinuidades por meio da função joint network. O software fornece como resultado um fator de resistência mínimo, que é análogo ao fator de segurança dos métodos analíticos. 


\section{CARACTERIZAÇÃO DA ÁREA ESTUDADA}

A mina de Candiota está localizada no município de Pinheiro Machado - RS e extrai calcário calcítico para a indústria de cimento. Os taludes finais da cava são compostos por xistos e filitos, que são as rochas encaixantes das lentes de calcário. Em um dos taludes globais finais, composto por filito, foi identificado potencialidade para ruptura por tombamento com outras quatro famílias de descontinuidades que mergulham em diferentes direções.

O mergulho das lentes de calcário varia entre 50 e 70 graus. A cava, conforme representado na Figura 1, possui geometria em forma de elipse alongada na direção da lente de calcário.

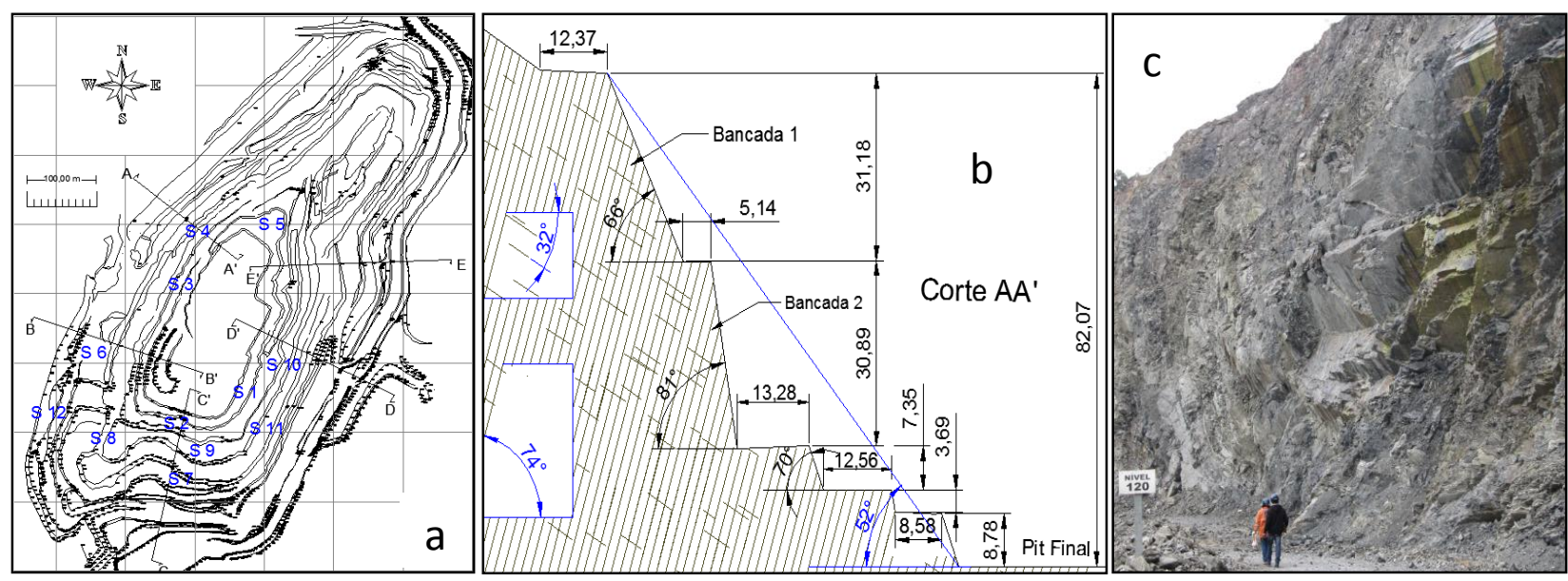

Figura 1: Layout da cava da mina com a localização das Scanlines (a); corte da secção AA' (b) e imagem do talude em situação de tombamento (c).

A Figura 1a mostra o layout da cava com os pontos onde houve mapeamento por Scanlines. A Figura $1 \mathrm{~b}$ representa a geometria do talude global no corte $A A^{\prime}$ com a representação das descontinuidades propícias a tombamento e a Figura 1 c revela a imagem do talude em situação de tombamento. Observa-se ainda que alguns desprendimentos de blocos rochas já ocorreram.

\section{METODOLOGIA}

\subsection{Análise cinemática}

Baseado em 12 medidas de Scanlines, as orientações das principais famílias de descontinuidades foram definidas conforme a Tabela 1.

Tabela 1: Orientação das famílias de descontinuidades

\begin{tabular}{cc}
\hline Família & Orientação \\
\hline $1 \mathrm{~m}-$ Foliação & $74 / 317$ \\
$2 \mathrm{~m}-$ Foliação & $34 / 316$ \\
$3 \mathrm{~m}$ & $72 / 053$ \\
$4 \mathrm{~m}$ & $32 / 098$ \\
$5 \mathrm{~m}$ & $69 / 156$ \\
\hline
\end{tabular}

A partir das orientações das descontinuidades em relação a face do talude, a análise cinemática auxiliada por meio do software Dips V.5 da Rocscience, constatou em um dos setores da cava, potenciais para rupturas por tombamento. Como demonstrado na Figura 2, a condição de 
instabilidade ocorreu porque os polos das descontinuidades, que mergulham para dentro da face do talude, estão dentro de $30^{\circ}$ do Dip Ditection da face do talude (Wyllie e Mah, 2004), isto foi representado pela densidade de polos.

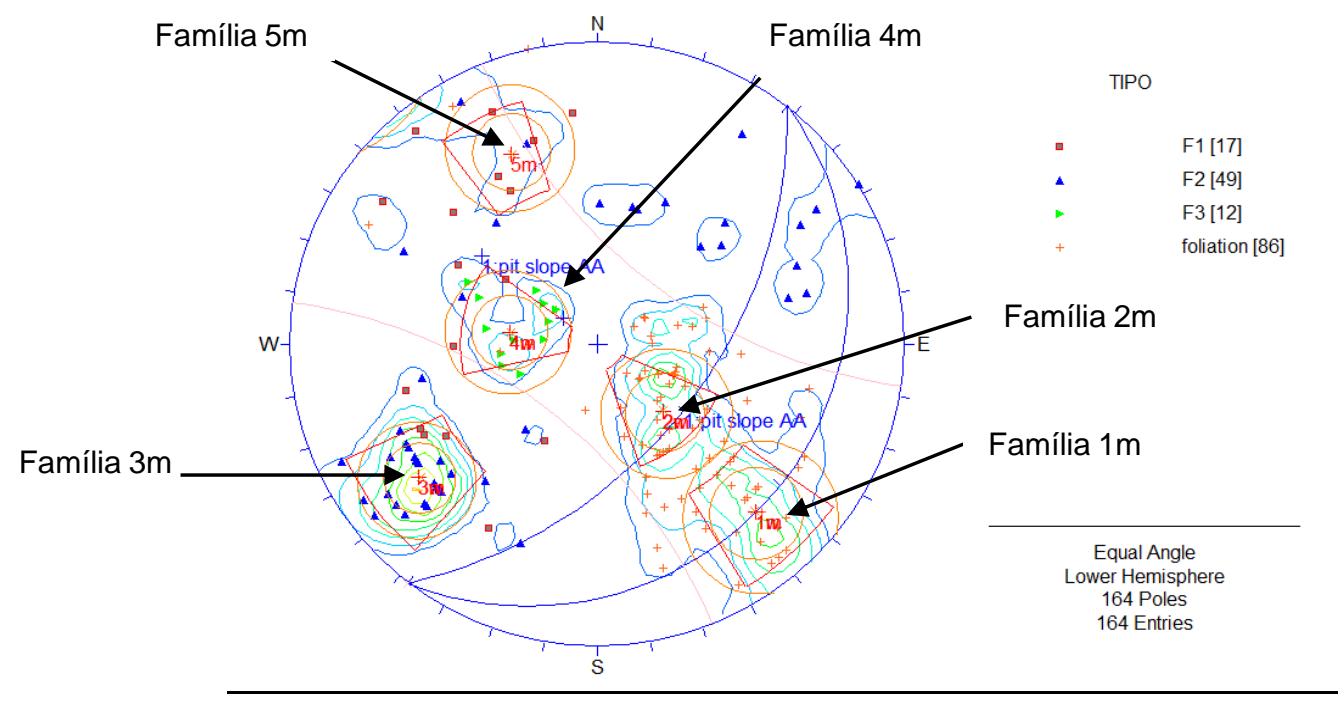

Figura 2: Análise cinemática com auxílio do Dips V.5

Observa-se que o talude $A A^{\prime}$ possui possibilidades de tombamento, condicionado pela densidade de foliação das famílias $1 \mathrm{~m}$ e $2 \mathrm{~m}$ nesse setor, com cerca de $40 \%$ das descontinuidades em condição para tombamento. A avaliação da estabilidade foi considerada para o talude global.

\subsection{Caracterização geomecânica}

Ensaios triaxiais, utilizados para obter os parâmetros de coesão e ângulo de atrito da rocha, foram tratados no software RocLab V.1 da Rocscience por meio do critério de Mohr-Coloumb (Wyllie e Mah, 2004). Embora nos ensaios triaxiais as tensões de confinamento ( $\sigma 3$ ) devam ser estimadas como a metade da resistência a compressão da rocha $\left(\sigma_{c i}\right)$ (Hoek e Brown, 1997) as tensões $\sigma 3$ foram consideradas conforme as tensões confinantes em subsuperfície, pois a profundidade máxima da cava é de 90,00 metros. A resistência a tração foi estimada como $10 \%$ de $\sigma_{\mathrm{ci}}$, o resultado dos ensaios triaxiais estão representados na Tabela 2.

Tabela 2: Resultados dos ensaios de compressão triaxial

\begin{tabular}{ccc}
\hline $\begin{array}{c}\text { Tensão Confinante } \sigma 3 \\
(\mathrm{MPa})\end{array}$ & $\begin{array}{c}\text { Tensão Axial } \sigma 1 \\
\text { (MPa) Calcário }\end{array}$ & $\begin{array}{c}\text { Tensão Axial } \sigma 1 \\
\text { (MPa) Filito }\end{array}$ \\
\hline 1.0 & 65.57 & 49.5 \\
2.0 & 71.86 & 53.8 \\
4.0 & 80.37 & 60.1 \\
\hline
\end{tabular}

Os parâmetros de resistência do maciço foram estimados por meio da integração dos resultados dos ensaios triaxiais e da classificação geomecânica GSI - Geological Strength Index (Hoek, Karranz e Torres, 2002), conforme Equação 3.

$\sigma 1=\sigma 3+\sigma c i\left(\mathrm{mb}\left(\frac{\sigma 3}{\sigma \mathrm{ci}}\right)+\mathrm{s}\right)^{\mathrm{a}}$ 
Onde oci é a resistência à compressão simples da rocha; $\mathrm{mb}$ é o valor reduzido da constante mi, definido pela Equação (4); as constantes s e a são definidas pelas equações (5) e (6); e D é o fator de perturbação em função do desmonte de rocha (Hoek, Carranza-Torres e Corkum, 2002).

$$
\begin{aligned}
& \mathrm{mb}=\mathrm{mi} \exp \left(\frac{\mathrm{GSI}-100}{28-14 \mathrm{D}}\right) \\
& \mathrm{s}=\exp \left(\frac{\mathrm{GSI}-100}{9-3 \mathrm{D}}\right) \\
& \mathrm{a}=\frac{1}{2}+\frac{1}{6}\left(\mathrm{e}^{-\mathrm{GSI} / 15}-\mathrm{e}^{-20 / 3}\right)
\end{aligned}
$$

Os parâmetros de resistência das descontinuidades foram estimados conforme sugerido por Barton e descrito em Hoek, Kaiser e Bawden (1995) por meio da Equação (7). A resistência a compressão das juntas (JCS) foram obtidos através do martelo de Schmidt com valor de $50 \mathrm{MPa}$. O coeficiente de rugosidade (JRC), estimado por observação (Barton, 1973), foi estipulado entre 4 e 10.

$\tau=\sigma \mathrm{n} \tan \left(\phi+\mathrm{JRC} \log 10\left(\frac{\mathrm{JCS}}{\sigma \mathrm{n}}\right)\right)$

Onde on é a tensão normal na descontinuidade e $\phi$ é o ângulo de atrito da descontinuidade. A tabela 3 resume os parâmetros de resistência obtidos para rocha e maciço. Os valores de coesão e ângulo de atrito das descontinuidades foram definidos em 0,057 e $40^{\circ}$, respectivamente.

Tabela 3: Parâmetros de resistência obtidos para o filito.

\begin{tabular}{ccc}
\hline Parâmetro & Rocha & Maciço \\
\hline GSI & 100 & 35 \\
Resistência oci (MPa) & 46.00 & 1.284 \\
Resistência ot (MPa) & 5.00 & 0,00 \\
Módulo de Elasticidade (MPa) & 18000 & 2086,60 \\
s (critério Hoek-Brown) & 1,0 & $1.9 \mathrm{e}-5$ \\
a (critério Hoek-Brown) & 0,5 & 0.516 \\
m (critério Hoek-Brown) & 5.699 & 0.559 \\
Coesão - c (MPa) & 10.78 & 0.126 \\
Ângulo de atrito interno $\phi$ & 36.45 & 21.86 \\
\hline
\end{tabular}

\subsection{Modelagem numérica}

A modelagem do problema baseou-se em definir os parâmetros necessários para a construção do modelo e verificar as deformações dos nós dos elementos segundo os critérios de Mohr-Coloumb e Hoek-Brown. O modelo foi construído utilizando os seguintes parâmetros: i) geometria da escavação, ii) geologia, iii) propriedades geomecânicas e estruturais do maciço rochoso, iv) estado de tensão e v) condições de borda do modelo.

Na mina de Candiota foram realizadas duas modelagens por elementos finitos. As análises utilizaram o critério de ruptura de Hoek e Brown (Hoek e Brown, 1997) com elementos triangulares de seis pontos nodais e as descontinuidades, quando inseridas no modelo, foram tratadas de acordo com o critério de Mohr-Coloumb. O estado de tensão para os dois modelos foram considerados como gravitacionais. 
A primeira modelagem tratou o maciço como um meio contínuo, elasto-plástico, homogêneo e com os parâmetros das descontinuidades inseridos na classificação geomecânica GSI (Hoek, Carranza-Torres e Corkum, 2002). Os parâmetros de entrada do modelo estão definidos na Tabela 4. Embora o modelo seja para maciço o valor de oci é um parâmetro de entrada no Phase2.

Tabela 4: Parâmetros de resistência utilizados na primeira modelage
\begin{tabular}{cc} 
Parâmetros do Maciço & Filito \\
\hline GSI & 35 \\
Resistência oci (MPa) & 46 \\
Módulo de Elasticidade - Ei (MPa) & $2.086,60$ \\
Poisson & 0,3 \\
s (critério Hoek-Brown) & 0,00073 \\
a (critério de Hoek-Brown) & 0,516 \\
mb (critério Hoek-Brown) & 0,559 \\
\hline
\end{tabular}

A Figura 3 mostra a malha utilizada para discretizar o problema na primeira situação, onde o modelo foi tratado como um meio equivalente contínuo.

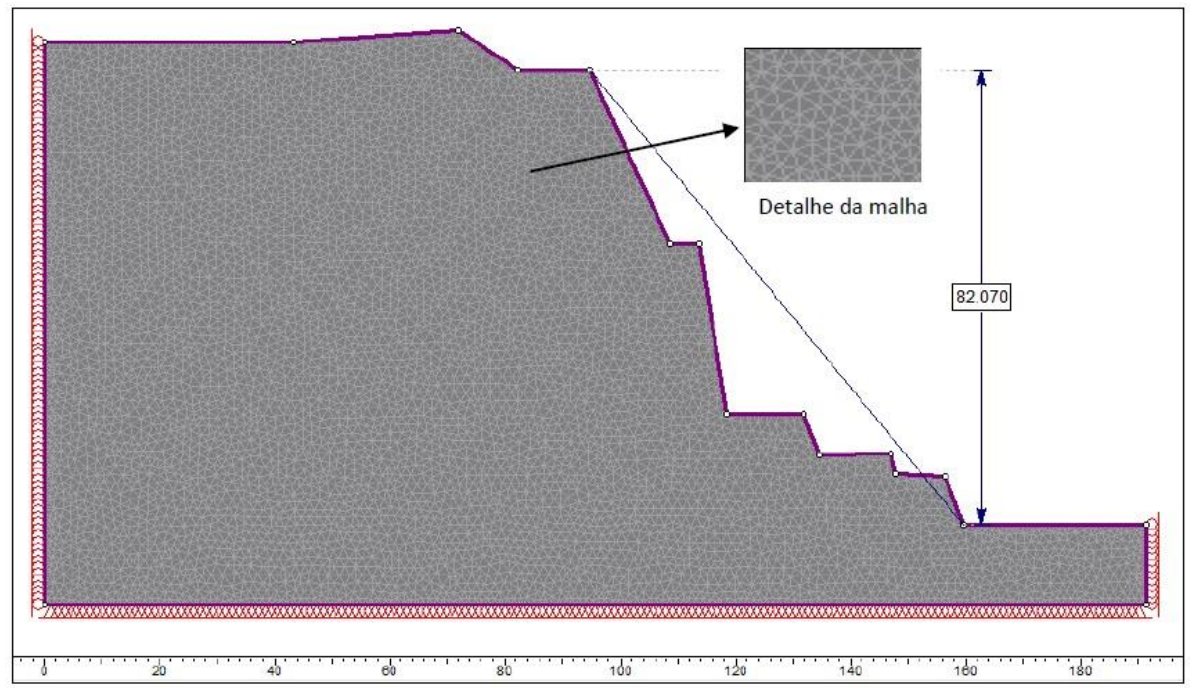

Figura 3: Discretização do modelo em malha triangular com 6 nós.

A segunda modelagem consistiu em discretizar o problema inserindo no modelo contínuo as descontinuidades, que possuem características capazes de contribuir para rotação ou flexão das colunas de rochas. Nesse modelo, o maciço foi tratado como um meio elástico e com características de rocha, uma vez que ao inserir as descontinuidades na rocha tentou-se obter a equivalência de características com o primeiro modelo, porém discretizadas pelas famílias de juntas.

As descontinuidades foram inseridas por meio da função joint net work e tratadas como elastoplásticas, pois a ruptura do talude deveria ocorrer nas zonas em que as tensões cisalhantes fossem maiores que a resistência ao cisalhamento das descontinuidades.

Nas Tabelas 5 e 6 estão definidos os parâmetros geomecânicos e estruturais da segunda modelagem, respectivamente. A Figura 4 mostra a discretização do problema com as descontinuidades inseridas no modelo contínuo. 
Tabela 5: Parâmetros de resistência utilizados na segunda modelagem

\begin{tabular}{cc}
\hline Parâmetros da Rocha (Hoek-Brown) & Filito \\
\hline GSI & 100 \\
Resistência oci (MPa) & 46 \\
Módulo de Elasticidade - Ei (MPa) & 18.000 \\
Poisson & 0,3 \\
s (critério Hoek-Brown) & 1,0 \\
a (critério de Hoek-Brown) & 0,5 \\
mi (critério Hoek-Brown) & 6 \\
\hline
\end{tabular}

Tabela 6: Características das descontinuidades inseridas no segundo modelo

\begin{tabular}{cccc}
\hline Família & $\begin{array}{c}\text { Orientação (dip/dip } \\
\text { direction) }\end{array}$ & $\begin{array}{c}\text { Espaçamento } \\
(\mathrm{m})\end{array}$ & $\begin{array}{c}\text { Persistência } \\
(\mathrm{m})\end{array}$ \\
\hline Foliação-1 & $74 / 317$ & 0,4 & 0,5 \\
Foliação-2 & $34 / 316$ & 1,0 & 0,5 \\
F1 & $69 / 156$ & 5,0 & 0,7 \\
F2 & $72 / 053$ & 4,0 & 0,5 \\
F3 & $32 / 098$ & 3,0 & 0,8 \\
\hline
\end{tabular}

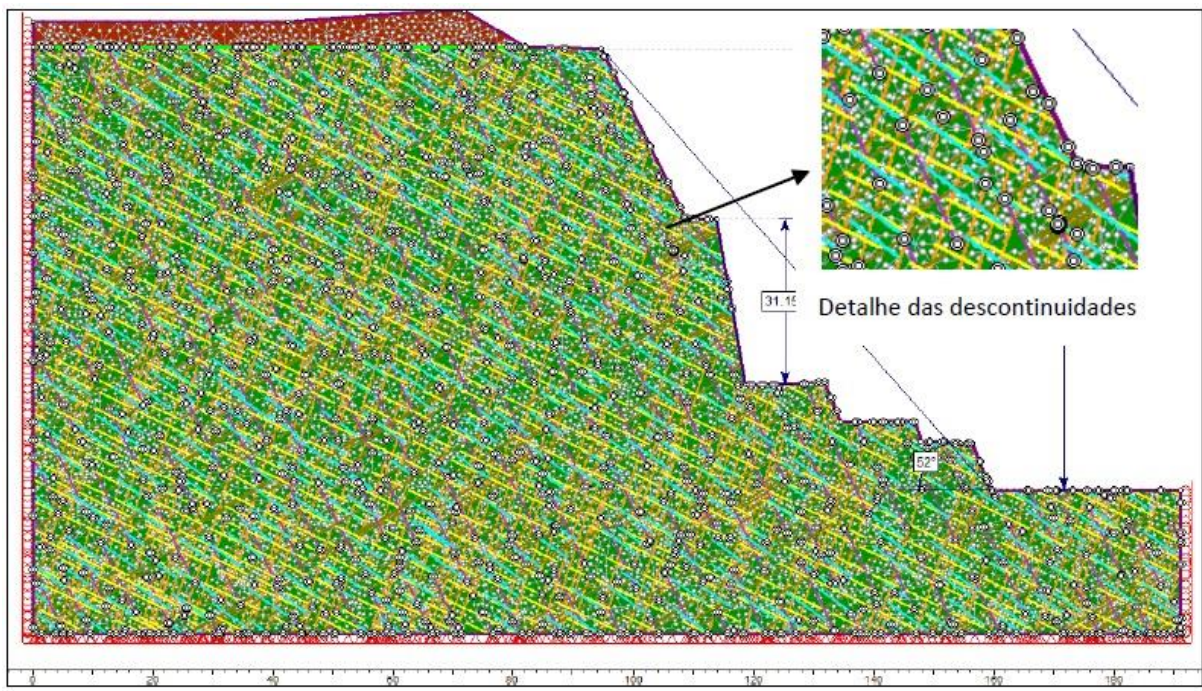

Figura 4: Discretização do modelo contínuo com as descontinuidades inseridas

\section{RESULTADOS}

A Figura 5 mostra o resultado do modelo contínuo equivalente simulado para os taludes finais da mina de Candiota. Observa-se condição de estabilidade para o ângulo global com fatores de resistência de 1,14. 


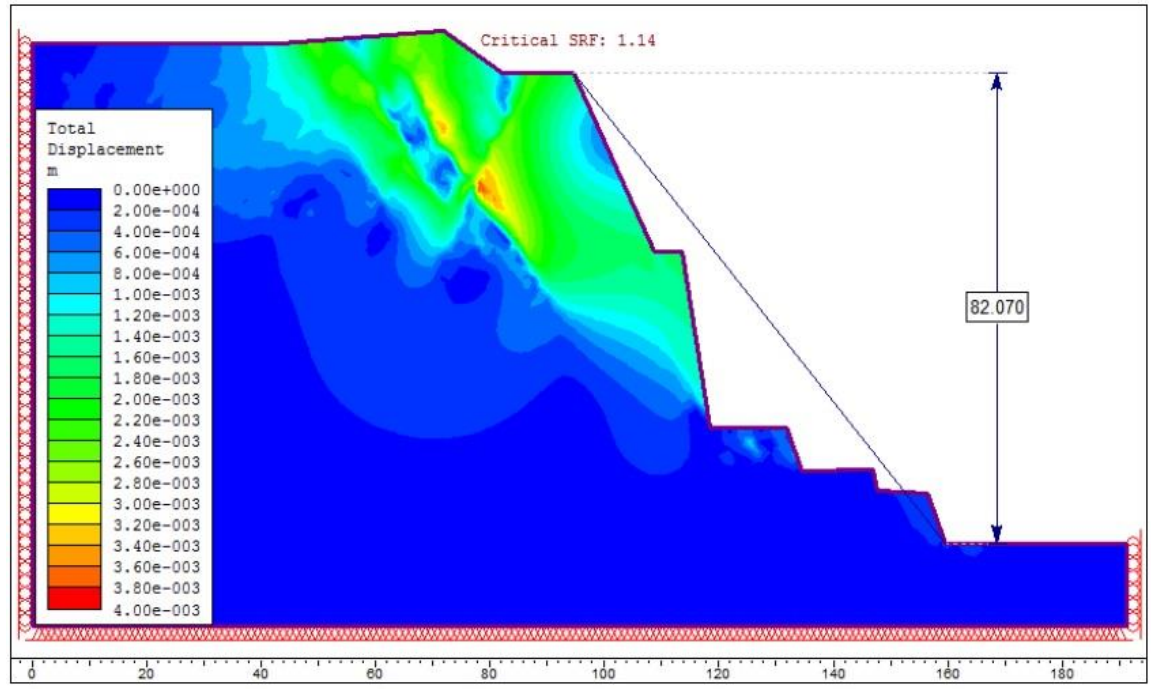

Figura 5: Modelo equivalente contínuo mostrando condições de estabilidade e fator de segurança de 1,14

O uso da função SRF, que é a redução progressiva das propriedades de resistência do maciço, com um exagero na escala através da função Display Deformation Contours, permitiu enxergar nas modelagens como tende a ser a ruptura do talude. No modelo continuo, Figura 6, observa-se a tendência de ruptura do talude como estrutura única não sendo possível enxergar o movimento de tombamento.

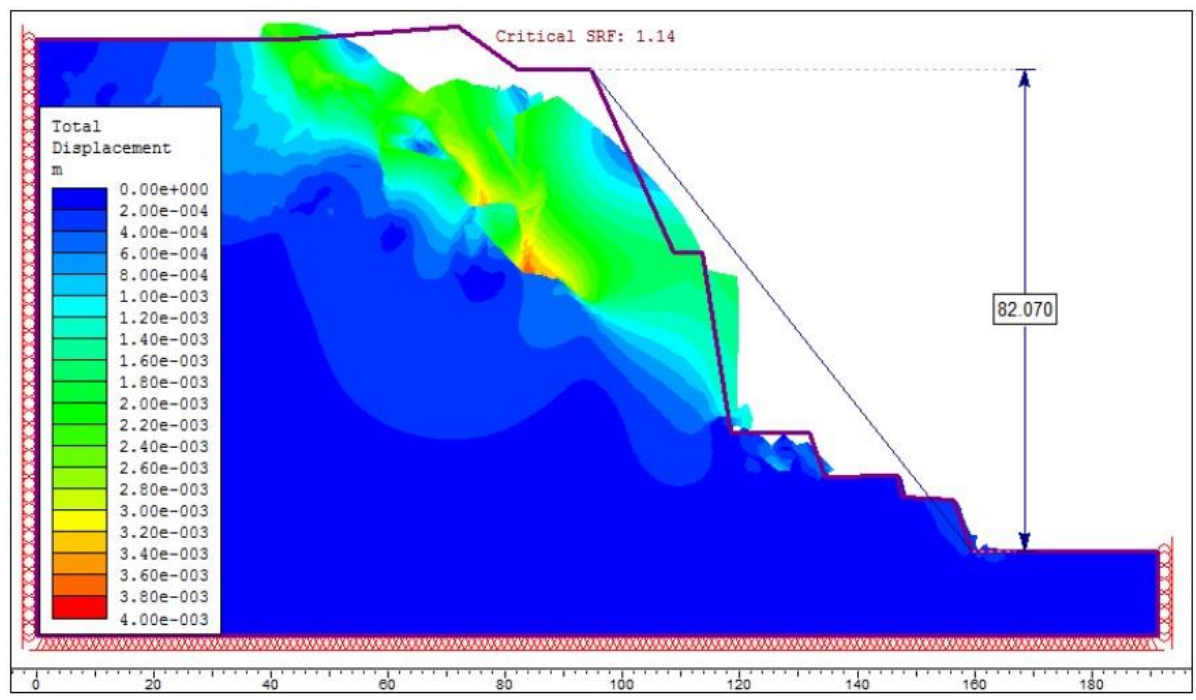

Figura 6: Modelo equivalente contínuo mostrando a ruptura do talude

No segundo modelo o fator de resistência estimado foi de 1,2. Os resultados dos dois modelos sugerem uma equivalência entre o modelo contínuo, com as descontinuidades inseridas, e o contínuo equivalente, uma vez que os fatores de segurança tiveram valores próximos.

Entretanto, a função Display Deformation Contours, aplicada ao segundo modelo, mostra o aparecimento de um plano basal com ruptura do talude nas descontinuidades, simulando o deslocamento do talude global por movimento de tombamento (Figura 7). No segundo caso, o tombamento ficou evidente devido as descontinuidades estarem explícitas no problema. 


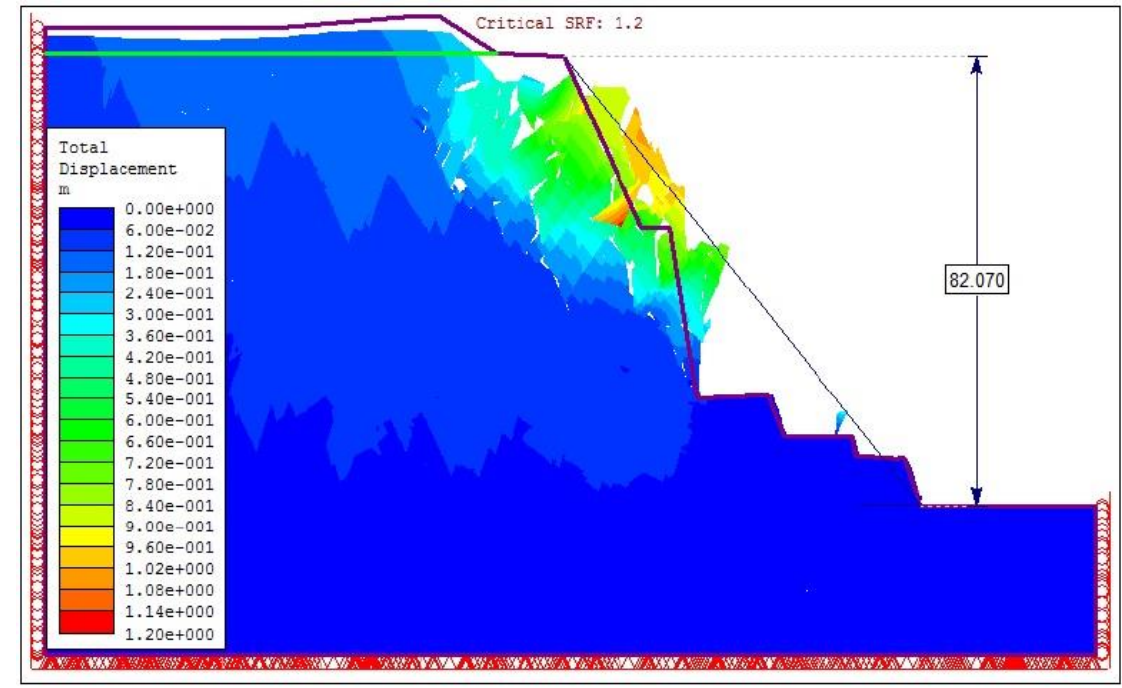

Figura 7: Modelo contínuo com as descontinuidades inseridas mostrando fator de resistência de 1,2 e simulando o tombamento do talude.

\section{CONCLUSÕES}

As duas modelagens mostraram-se eficientes ao tratar o maciço como um meio contínuo, por meio da classificação geomecânica, ou equivalentes contínuos, por meio inserção das descontinuidades no problema. Os resultados dos fatores de segurança em 1,14 e 1,2 estão próximos e em níveis de estabilidade. Embora haja similaridade nos fatores de segurança das duas modelagens só foi possível enxergar o movimento de tombamento no problema discretizado, com a ruptura ocorrendo nas descontinuidades.

\section{REFERÊNCIAS BIBLIOGRÁFICAS}

1. ADHIKARY, D. P.; DYSKIN, A. V.; JEWELL, R. J. Numerical Modelling of the Flexural Deformation of Foliated Rock Slopes. Int. J. Rock Mech. Min. Sci. \& Geomech. V. 33, p. 595 - 606, 1996.

2. ADHIKA RY, D. P.; DYSKIN, A. V.; JEWELL, R. J.; STEWART, D. P. A Study of the Mechanism of Flexural Toppling Failure of Rock Slopes. Rock Mech. Rock Engng. V.30 (2), p. 75 - 93, 1997.

3. AMINI, M.; MAJDI, A.; VESHADI, M. Stability Analysis of Rock Slopes Against Block-Flexure Toppling Failure. Rock Mech. Rock Engng. V. 45 (4), p. 519 - 532, 2012.

4. AMINI, M.; MAJDI, A.; AYDAN, O. Stability Analysis and the Stabilisation of Flexural Toppling Failure. Rock Mech. Rock Engng. V. 42 (5), p. 751 - 782, 2009.

5. AYDAN, O.; KAWAMOTO, T. The Stability of Slopes and Underground Openings Against Flexural Toppling and Their Stabilisation. Rock Mech. Rock Engng. V. 25 (3), p. 143 - 165, 1992.

6. BARTON, N. R. Review of a New Shear Strength Criterion for Rock Joints. Eng. Geol., Elsevier. V.7, p. $287-322,1973$.

7. BOBET, A. Analytical Solutions for Toppling Failure. Int. J. Rock Mech. Min. Sci. \& Geomech. V. 36, p. 971 - 980, 1999. 
8. BRADY, B. H. G.; BROWN, E. T. Rock Mechanics for Underground Mining. 3rd ed. New York: Springer Science and Business Media, 2004. 628 p.

9. BURMAN, B.C.; TROLOPE, D. H.; PHILIP, M. G. The Behaviour of Excavated Slopes in Jointed Rock. Autralian Geomechanics Journal. V. 12, p. $26-31,1975$.

10. GOODMAN, R.E.; BRAY, J.W. Toppling of Rock Slopes. In: ASCE speciality conference on rock engineering for foundation sands lopes, Boulder, Colorado; V. 2, p.201 - 34, 1976.

11. HOEK, E.; KAISER, P. K.; BAWDEN, W. F. Support of Underground Excavations in Hard Rock. 1st ed. Rotterdam: Balkema, 1995. 225 p.

12. HOEK, E.; BROWN, E.T. Practical Estimates of Rock Mass Strength. Int. J. Rock Mech. Min. Sci. \& Geomech. Abstr. V. 34 (8), p. $1165-1186,1997$.

13. HOEK, E.; CARRANZA-TORRES, C.; CORKUM, B. Hoek-Brown Criterion - 2002. In: Proceedings of the North American rock mechanics symposium, 5., 2002, Toronto, 2002. p. $267-273$.

14. HUDSON, J. A.; HARRISON, J. P. Engineering Rock Mechanics - an Introduction to the Principles. 1st ed. Londres: Pergamon press, 1997. 444 p.

15. LIU, C. H.; JAKSA, M. B.; MEYERS, A. G. (2009). A Transfer Coefficient Method for Rock Slope Toppling. Can.Geotech. J. V. 46, 1-9, 2009.

16. PEREIRA, L. C. Análise Tensão-deformação e por Equilíbrio Limite do Mecanismo de Ruptura por Tombamento de blocos. 2012. 184 pág. Dissertação (Mestrado) - Univ. Fed. de Ouro Preto. Escola de Minas. Ouro Preto, 2012.

17. PRITCHARD, M. Numerical Modelling of Large Scale Toppling 1989. 178 p. MSc. thesis. The University of British Columbia, Vancouver, 1989.

18. PRITCHARD, M. A.; SAVIGNY, K. W. Numerical Modelling of Toplling. Canadian Geotechnical Journal. V. 27, p. $823-834,1990$.

19. SAGASETA, C.; SÀNCHEZ, J.M.; CAÑIZAL, J. A general Analytical Solution for the Required Anchor Force in Rock Slopes with Toppling Failure. Int. J. Rock Mech. Min. Sci. \& Geomech. Abstr. V. 38, p. $421-435,2001$.

20. SANTOS, G. A. Análise Tensão-deformação e por Equilíbrio Limite do Mecanismo de Tombamento Flexural. 2009. 114 pág. Dissertação (Mestrado) - Univ. Fed. de Ouro Preto. Escola de Minas. Ouro Preto, 2009.

21. WYLLIE, D.C.; MAH, C.W. Rock Slope Engineering: Civil and Mining. 4th ed. London: UK Spon Press, 2004. 425 p.

22. ZANBAK, C. Design Charts for Rock Slopes Susceptible to Toppling. J Geotech Eng ASCE. V. 109 (8), p. $1039-1062,1983$. 